\title{
A SHORT SOLUTION OF MARKOV'S PROBLEM ON CONNECTED GROUP TOPOLOGIES
}

\author{
DIETER REMUS
}

(Communicated by Dennis Burke)

\begin{abstract}
In 1945 A. A. Markov posed a problem concerning the existence of connected group topologies that was recently solved by V. G. Pestov. In this note, a short and independent solution is given.
\end{abstract}

All topological groups are assumed to be Hausdorff. For a Russian article, the cited translation is used.

In [4] A. A. Markov discussed the existence of connected group topologies. He called a subset $M$ of an abstract group $G$ unconditionally closed in $G$ if $M$ is closed with respect to any group topology on $G$. (Note that this property is called "absolutely closed" in [5].) The following fact can be shown easily [4, Theorem 2.9, p. 270]: If a group $G$ has an unconditionally closed proper subgroup of index less than $2^{\aleph_{0}}$, then $G$ does not admit a connected group topology.

Now Markov asked whether the inverse theorem is true [4, Problem 5, p. 271]. Recently V. G. Pestov [5] has answered this old question in the negative. For the construction of his counterexample, Pestov develops a complicated technique of independent interest (cf. [5, $\S \S 1$ and 2]). In this note, quite different examples are given independently by a short proof (see theorem). In March 1989 the author reported on this result at Sofia (Bulgaria). Two weeks later, D. Dikranjan informed him of Pestov's paper.

Let $M$ be an infinite set. Then $S(M)$ denotes the group of all bijections from $M$ onto $M . S(M)$ is called the symmetric group of $M$.

Theorem. Let $M$ be a set with $|M| \geq 2^{\aleph_{0}}$. Then every unconditionally closed proper subgroup of $S(M)$ is of index not less than $2^{\aleph_{0}}$, but $S(M)$ admits only totally disconnected group topologies.

Proof. By [2] $S(M)$ has no proper subgroups of index less than $|M|$. Let $\tau_{M}$ be the topology of pointwise convergence on $S(M)$ with respect to the discrete topology on $M$. It is easy to see that $\tau_{M}$ is a group topology which has, as a subbasis for the neighbourhoods of the identity, the collection of all subgroups

Received by the editors July 6, 1989 and, in revised form, December 11, 1989.

1980 Mathematics Subject Classification (1985 Revision). Primary 22A05; Secondary 54D05. 
of the form $E_{x}=\{f \in S(M): f(x)=x\}$, where $x \in M$ (cf. [1, §7.1]). Then [3, Theorem 2] implies that on $S(M)$ every group topology $\tau$ is finer than $\tau_{M}$. Since $\tau_{M}$ is totally disconnected, $\tau$ has the same property.

It is natural to pose the following:

Question. Is there an abelian group $G$ such that $G$ does not admit a connected group topology, but every unconditionally closed proper subgroup of $G$ is of index not less than $2^{\aleph_{0}}$ ?

\section{REFERENCES}

1. D. Dikranjan, Iv. Prodanov, and L. Stojanov, Topological groups: characters, dualities and minimal group topologies, Pure and Applied Math., Marcel Dekker, New York, 1989.

2. E. D. Gaughan, The index problem for infinite symmetric groups, Proc. Amer. Math. Soc. 15 (1964), 527-528.

3. $\ldots$, Topological group structures of infinite symmetric groups, Proc. Nat. Acad. Sci. U. S. A. 58 (1967), 907-910.

4. A. A. Markov, On free topological groups, Izv. Akad. Nauk SSSR, Ser. Mat. 9 (1945), 3-64 (Russian); English transl., Amer. Math. Soc. Transl. Ser. 18 (1962), 195-272.

5. V. G. Pestov, Absolutely closed sets and a hypothesis of A. A. Markov, Sibirsk. Mat. Zh. 29 (1988), 124-132 (Russian); English transl., Siberian Math. J. 29 (1988), 260-266.

Institut für Mathematik, Lehrgebiet D, Universität Hannover, D-3000 Hannover, Federal Republic of Germany 